\title{
Multiplicity of solutions for a nonhomogeneous problem involving a potential in Orlicz-Sobolev spaces
}

\section{Multiplicité de solutions pour un problème non homogène impliquant un potentiel dans les espaces d'Orlicz-Sobolev}

\author{
Nawal Irzi
}

Mathematic Department, Faculty of Science of Tunis, University of Tunis El Manar 1060, Tunis, Tunisia

nawalirzi15@gmail.com

ABSTRACT. This paper is devoted to the study of the nonhomogeneous problem $-\operatorname{div}(a(|\nabla u|) \nabla u)+a(|u|) u=$ $\lambda V(x)|u|^{m(x)-2} u-\mu g(x, u)$ in $\Omega, u=0$ on $\partial \Omega$, where $\Omega$ is a bounded smooth domain in $\mathbb{R}^{N}, \lambda, \mu$ are positive real numbers, $V(x)$ is a potential, $m: \bar{\Omega} \rightarrow(1, \infty)$ is a continuous function, $a$ is mapping such that $\varphi(|t|) t$ is increasing homeomorphism from $\mathbb{R}$ to $\mathbb{R}$ and $g: \bar{\Omega} \times \mathbb{R} \rightarrow \mathbb{R}$ is a continuous function. We establish there main results with various assumptions, the first one asserts that any $\lambda>0$ is an eigenvalue of our problem. The second Theorem states the existence of a constant $\lambda^{*}$ such that every $\lambda \in\left(0, \lambda^{*}\right)$ is an eigenvalue of the problem. While the third Theorem claims the existence of a constant $\lambda^{* *}$ such that every $\lambda \in\left[\lambda^{* *}, \infty\right)$ is an eigenvalue of the problem. Our approach relies on adequate variational methods in Orlicz-Sobolev spaces.

2010 Mathematics Subject Classification. 35J65, 35D05, 35J60, 35D30, 35J58.

KEYWORDS. Mountain pass Theorem, Ekeland's variational principle, Orlicz-Sobolev space.

\section{Introduction}

We are interested here with the existence of solutions for a nonhomogeneous problem in Orlicz-Sobolev spaces

$$
\begin{cases}-\operatorname{div}(a(|\nabla u(x)|) \nabla u(x))+a(|u(x)|) u(x)=\lambda V(x)|u(x)|^{m(x)-2} u(x)-\mu g(x, u(x)) & \text { in } \Omega \\ u(x)=0 & \text { on } \partial \Omega,\end{cases}
$$

where, $\Omega$ is a bounded domain in $\mathbb{R}^{N}$ with smooth boundary, $(N \geq 3), \lambda, \mu$ are positive real numbers, the potential $V(x): \Omega \rightarrow \mathbb{R}$ satisfies $V \in L^{r(x)}(\Omega)$ with $r, m: \bar{\Omega} \rightarrow(1, \infty)$ are continuous functions and $g: \bar{\Omega} \times \mathbb{R} \rightarrow \mathbb{R}$ is a continuous function where will be imposed some suitable conditions later. Assume that $a:(0, \infty) \rightarrow \mathbb{R}$ is a function such that the mapping $\varphi: \mathbb{R} \rightarrow \mathbb{R}$, defined by

$$
\varphi(t)= \begin{cases}a(|t|) t & t \neq 0 \\ 0 & t=0\end{cases}
$$

is an odd, strictly increasing homeomorphism from $\mathbb{R}$ onto $\mathbb{R}$.

It should be noticed that if $a(t)=|t|^{p(x)-2}$, with $p(x)$ a continuous function on $\bar{\Omega}$, then problem (1.1) becomes the well-known Dirichlet boundary value problem involving the $p(x)$-Laplacian equation

$$
\begin{cases}-\Delta_{p(x)} u(x)+|u(x)|^{p(x)-2} u(x)=\lambda V(x)|u(x)|^{m(x)-2} u(x)-\mu g(x, u(x)) & \text { in } \Omega \\ u(x)=0 & \text { on } \partial \Omega .\end{cases}
$$

In recent years, this type of problem has been largely considered in many papers, we refer to $[5,9,15,17]$ in which the authors have used different methods to prove the existence and multiplicity of solutions. 
Many researchers have studied the existence of solutions for eigenvalue problems involving nonhomogeneous operators in the divergence form in Orlicz- Sobolev spaces by means of variational methods, see for examples [3, 19, 20, 21]. In [8] Clément et al. established the existence of weak solutions for the following Dirichlet problem

$$
\begin{cases}-\operatorname{div}(a(|\nabla u(x)|) \nabla u(x))=g(x, u(x)) & \text { in } \Omega \\ u(x)=0 & \text { on } \partial \Omega,\end{cases}
$$

where $\Omega$ is a bounded domain in $\mathbb{R}^{N}, g \in C(\bar{\Omega} \times \mathbb{R}, \mathbb{R})$ and the function $\varphi(s)=s a(|s|)$ is an increasing homeomorphism from $\mathbb{R}$ onto $\mathbb{R}$. Under appropriate conditions on $\varphi, g$ and the Orlicz-Sobolev conjugate $\Phi^{*}$ of $\Phi(s)=\int_{0}^{s} \varphi(t) d t$, they obtained the existence of non-trivial solutions of mountain pass type. Moreover, Clément et al. in [7] used Orlicz-Sobolev spaces theory and a variant of the Mountain Pass Lemma of Ambrosetti-Rabinowitz to obtain the existence of a (positive) solution to a semi-linear system of elliptic equations. In addition, by adequate variational methods in Orlicz-Sobolev spaces, Mihăilescu and Rădulescu [20] studied the boundary value problem

$$
\begin{cases}-\operatorname{div}\left(\left(a_{1}(|\nabla u(x)|)+a_{2}(|\nabla u(x)|)\right) \nabla u(x)\right)=\lambda|u(x)|^{q(x)-2} u(x) & \text { in } \Omega \\ u(x)=0 & \text { on } \partial \Omega,\end{cases}
$$

where $\Omega$ is a bounded domain in $\mathbb{R}^{N}(N \geq 3)$ with smooth boundary, $\lambda$ is a positive real number, $q$ is a continuous function and $a_{1}, a_{2}$ are two mappings such that $a_{1}(|t|) t, a_{2}(|t|) t$ are increasing homeomorphisms from $\mathbb{R}$ to $\mathbb{R}$. They established the existence of two positive constants $\lambda_{0}$ and $\lambda_{1}$ with $\lambda_{0} \leq \lambda_{1}$ such that any $\lambda \in\left[\lambda_{1}, \infty\right)$ is an eigenvalue, while any $\lambda \in\left(0, \lambda_{0}\right)$ is not an eigenvalue of the problem (1.3).

Mihăilescu and Repovš in [19], by combining Orlicz-Sobolev spaces theory with adequate variational methods and a variant of Mountain Pass Lemma, proved the existence of at least two non-negative and non-trivial weak solutions for the problem

$$
\begin{cases}-\operatorname{div}(a(|\nabla u(x)|) \nabla u(x))=\lambda f(x, u(x)) & \text { in } \Omega \\ u(x)=0 & \text { on } \partial \Omega,\end{cases}
$$

where $a$ is the same as in the problem (1.1), $f: \Omega \times \mathbb{R} \rightarrow \mathbb{R}$ is a Carathéodory function and $\lambda$ is a positive parameter.

In this paper, we analyze problem (1.1) under the following basic assumptions

$\left(A_{1}\right) g: \bar{\Omega} \times \mathbb{R} \longrightarrow \mathbb{R}$ is a continuous function satisfies

$$
|g(x, t)| \leq C_{0}|t|^{q(x)-1} \forall x \in \Omega, t \in \mathbb{R},
$$

and

$$
C_{1}|t|^{q(x)} \leq G(x, t)=\int_{0}^{t} g(x, s) d s \leq C_{2}|t|^{q(x)} \forall x \in \Omega, t \in \mathbb{R},
$$

where $C_{0}, C_{1}$ and $C_{2}$ are positive constants and $q(x) \in C(\bar{\Omega})$. 
$\left(A_{2}\right)$ The potential $V: \bar{\Omega} \rightarrow \mathbb{R}$ satisfies

$$
V \in L^{r(x)}(\Omega) \text {, with } r(x) \in C(\bar{\Omega}) \text { and } r(x)>N, \forall x \in \bar{\Omega} .
$$

We notice that the operator in the divergence form is not homogeneous and thus, we introduce an Orlicz-Sobolev space setting for problems of this type. On the other hand, the presence of the continuous exponent functions $m, r$ and $q$ appeals to a suitable variable exponent Lebesgue space setting. We should note that, as the Orlicz spaces, denoted by $L_{\phi}(\Omega)$, are a generalization of the Lebesgue spaces $L^{p}(\Omega)$, so the Orlicz-Sobolev spaces, denoted by $W^{m} L_{\phi}(\Omega)$, are a generalization of the Sobolev spaces $W^{m, p}(\Omega)$. Consequently, several properties of Sobolev spaces have been extended to Orlicz-Sobolev spaces. The Orlicz-Sobolev spaces are used to model various phenomena among which are the image restoration (see [6] ) and modeling of electrorheological fluids (see [26]). Both applications are based on variable exponent type Laplace operators.

The remainder of the paper is organized as follows. In the second section we make a brief introduction for each of the following spaces: Orlicz spaces, Orlicz-Sobolev spaces and variable exponent Lebesgue spaces. In the third section, we give some results of the existence of solution for problem (1.1) and by adequate variational methods, we prove this results in Orlicz-Sobolev spaces.

\section{Terminology and abstract setting}

We firstly recall some basic facts about Orlicz spaces. For more details, we refer to the books by D.R. Adams, L.I. Hedberg [1], R. Adams [2], M.M. Rao, Z.D. Ren [25] and the papers by Ph. Clément et al. [7], M. Garciá-Huidobro et al. [13].

For $\varphi: \mathbb{R} \rightarrow \mathbb{R}$, which is odd, increasing homeomorphism from $\mathbb{R}$ onto $\mathbb{R}$, we define

$$
\Phi(t)=\int_{0}^{t} \varphi(s) d s, \quad(\Phi)^{*}(t)=\int_{0}^{t}(\varphi)^{-1}(s) d s, \quad \text { for all } t \in \mathbb{R} .
$$

We observe that $\Phi$ is Young function, that is, $\Phi(0)=0, \Phi$ is convex and $\lim _{x \rightarrow+\infty} \Phi(x)=+\infty$. Furthermore, since $\Phi(x)=0$, if and only if $x=0, \lim _{x \rightarrow 0} \frac{\Phi(x)}{x}=0$ and $\lim _{x \rightarrow+\infty} \frac{\Phi(x)}{x}=+\infty$, then $\Phi$ is called N-function. The function $(\Phi)^{*}$, is called the complementary function of $\Phi$ and it satisfies

$$
(\Phi)^{*}(t)=\sup \{s t-\Phi(s) ; s \geq 0\}, \text { for all } t \geq 0 .
$$

We also observe that $(\Phi)^{*}$ is also $\mathrm{N}$-function and Young's inequality holds true

$$
s t \leq \Phi(s)+(\Phi)^{*}(t), \quad \text { for all } s, t \geq 0 .
$$

The Orlicz spaces $L_{\Phi}(\Omega)$ defined by the $\mathrm{N}$-function $\Phi$ are the spaces of measurable functions $u: \Omega \rightarrow$ $\mathbb{R}$ such that

$$
\|u\|_{L_{\Phi}}:=\sup \left\{\int_{\Omega} u v d x ; \int_{\Omega}(\Phi)^{*}(|v|) d x \leq 1\right\}<\infty .
$$

Then $\left(L_{\Phi}(\Omega),\|u\|_{L_{\Phi}}\right)$ is a Banach space whose norm is equivalent to the Luxemburg norm

$$
\|u\|_{\Phi}:=\inf \left\{k>0 ; \int_{\Omega} \Phi\left(\left|\frac{u(x)}{k}\right|\right) \leq 1\right\} .
$$


For Orlicz spaces Holder's inequality reads as follows (see [25])

$$
\int_{\Omega} u v d x \leq 2\|u\|_{L_{\Phi}}\|v\|_{L_{(\Phi) *}} \quad \text { for all } u \in L_{\Phi}(\Omega) \text { and } v \in L_{(\Phi)^{*}}(\Omega) .
$$

We denote by $W^{1} L_{\phi}$, the Orlicz-Sobolev space defined by

$$
W^{1} L_{\Phi}(\Omega)=\left\{u \in L_{\Phi}(\Omega) ; \frac{\partial u}{\partial x_{i}} \in L_{\Phi}(\Omega), i=1, \ldots, N\right\}
$$

This space endowed with the norm

$$
\|u\|_{1, \Phi}=\|u\|_{\Phi}+\|\mid \nabla u\|_{\Phi}
$$

is a reflexive Banach space. We also define the Orlicz-Sobolev space $W_{0}^{1} L_{\Phi}(\Omega)$, as the closure of $C_{0}^{\infty}(\Omega)$ in $W^{1} L_{\Phi}(\Omega)$. By Lemma 5.7 in [14] we obtain that on $W_{0}^{1} L_{\Phi}(\Omega)$ we may consider an equivalent norm

$$
\|u\|=\|\mid \nabla u\|_{\Phi}
$$

The space $W_{0}^{1} L_{\Phi}(\Omega)$ is also a reflexive Banach space.

We define

$$
\varphi_{0}:=\inf _{t>0} \frac{t \varphi(t)}{\Phi(t)} \text { and } \varphi^{0}:=\sup _{t>0} \frac{t \varphi(t)}{\Phi(t)},
$$

and we assume that we have

$$
1<\varphi_{0} \leq \frac{t \varphi(t)}{\Phi(t)} \leq \varphi^{0}<\infty \quad \forall t \geq 0 .
$$

The above relation implies that each $\Phi$, satisfies the $\Delta_{2}$-condition, i.e.

$$
\Phi(2 t) \leq K \Phi(t), \quad \forall t \geq 0
$$

where $K$ is a positive constant (see Proposition 2.3, [18]).

Furthermore, we assume that $\Phi$ satisfies the following condition

for each $x \in \bar{\Omega}$, the function $[0, \infty) \ni t \rightarrow \Phi(\sqrt{t})$ is convex.

Remark 1. Relations (2.3) and (2.4) assure that the Orlicz space $L_{\Phi}(\Omega)$ is an uniformly convex space and thus, a reflexive Banach space (see Proposition 2.2, [18]). That fact implies that also the Orlicz-Sobolev space $W_{0}^{1} L_{\Phi}(\Omega)$ is a reflexive Banach space.

We also recall the following Lemma which will be used in the proofs.

Lemma 2.1. (see [16], Lemma 2.3) Let $u \in W^{1} L_{\phi}(\Omega)$. Then

$$
\begin{aligned}
& \int_{\Omega}[\Phi(|u(x)|)+\Phi(|\nabla u(x)|)] d x \geq\|u\|^{\varphi^{0}}, \quad \text { if } \quad\|u\|<1, \\
& \int_{\Omega}[\Phi(|u(x)|)+\Phi(|\nabla u(x)|)] d x \geq\|u\|^{\varphi_{0}}, \quad \text { if } \quad\|u\|>1,
\end{aligned}
$$




$$
\begin{aligned}
& \int_{\Omega}[\Phi(|u(x)|)+\Phi(|\nabla u(x)|)] d x \leq\|u\|^{\varphi_{0}}, \quad \text { if } \quad\|u\|<1, \\
& \int_{\Omega}[\Phi(|u(x)|)+\Phi(|\nabla u(x)|)] d x \leq\|u\|^{\varphi^{0}}, \quad \text { if } \quad\|u\|>1 .
\end{aligned}
$$

Remark 2. We also note that with the help of condition (2.2), Orlicz-Sobolev space $W_{0}^{1} L_{\phi}(\Omega)$ is continuously embedded in the classical Sobolev space $W_{0}^{1, \varphi_{0}}(\Omega)$. On the other hand, $W_{0}^{1, \varphi_{0}}(\Omega)$ is compactly embedded in $L^{h(x)}(\Omega)$, as a result, $W_{0}^{1} L_{\phi}(\Omega)$ is continuously and compactly embedded in the classical Lebesgue space $L^{h(x)}(\Omega)$ for all $1 \leq h(x)<\varphi_{0}^{*}:=\frac{N \varphi_{0}}{N-\varphi_{0}}$.

Example 1. We can give certain examples of functions $\varphi: \mathbb{R} \rightarrow \mathbb{R}$ which are odd, increasing homeomorphisms from $\mathbb{R}$ onto itself and satisfy conditions (2.2) and (2.4):

- Let

$$
\varphi(t)=p|t|^{p-2} t, \forall t \in \mathbb{R},(\text { with } p>1) .
$$

For this function it can be proved that

$$
(\varphi)_{0}=(\varphi)^{0}=p
$$

Furthermore, in this particular case the corresponding Orlicz space $L_{\Phi}(\Omega)$ is the classical Lebesgue space $L^{p}(\Omega)$ while the Orlicz-Sobolev space $W_{0}^{1} L_{\phi}(\Omega)$ is the classical Sobolev space $W_{0}^{1, p}(\Omega)$. We will use the classical notations to denote the Orlicz-Sobolev spaces in this particular case.

- Consider

$$
\varphi(t)=\log \left(1+|t|^{s}\right)|t|^{p-2} t, \quad \forall t \in \mathbb{R},(\text { with } s, p>1) .
$$

In this case it can be proved that

$$
(\varphi)_{0}=p,(\varphi)^{0}=p+s .
$$

- Let

$$
\varphi(t)=\frac{|t|^{p-2} t}{\log (1+|t|)} \text { if } t \neq 0, \varphi(0)=0(\text { with } p>2) .
$$

In this case we have

$$
(\varphi)_{0}=p-1, \quad(\varphi)^{0}=p
$$

Next, we introduce some results and basic properties of the variable exponent Lebesgue space and Sobolev space $L^{p(x)}(\Omega), W^{k, p(x)}(\Omega)$, For further information in this regards we refer to [12, 23, 24].

Set

$$
C_{+}(\bar{\Omega}):=\{h: h \in C(\bar{\Omega}), h(x)>1, \text { for all } x \in \bar{\Omega}\} .
$$

For any $p \in C_{+}(\bar{\Omega})$, we denote

$$
1<p^{-}:=\min _{x \in \bar{\Omega}} p(x) \leq p^{+}=\max _{x \in \bar{\Omega}} p(x)<\infty .
$$


We define the variable exponent Lebesgue space by

$$
L^{p(x)}(\Omega)=\left\{u: \Omega \rightarrow \mathbb{R} \text { measurable and } \int_{\Omega}|u(x)|^{p(x)} d x<\infty\right\} .
$$

The space $L^{p(x)}(\Omega)$ is endowed with the Luxemburg norm, which is defined by

$$
|u|_{p(x)}=\inf \left\{\mu>0: \int_{\Omega}\left|\frac{u(x)}{\mu}\right|^{p(x)} d x \leq 1\right\} .
$$

We remember that the variable exponent Lebesgue spaces are separable and reflexive Banach spaces. If $0<|\Omega|<\infty$ and $q_{1}, q_{2}$ are variable exponents so that $q_{1}(x) \leq q_{2}(x)$ almost everywhere in $\Omega$ then there exists the continuous embedding $L^{q_{2}(x)}(\Omega) \hookrightarrow L^{q_{1}(x)}(\Omega)$.

Clearly, when $p(x) \equiv p$, the space $L^{p(x)}(\Omega)$ reduces to the classical Lebesgue space $L^{p}(\Omega)$ and the norm $|u|_{p(x)}$ reduces to the standard norm $\|u\|_{L^{p}}=\left(\int_{\Omega}|u|^{p} d x\right)^{\frac{1}{p}}$ in $L^{p}(\Omega)$.

Let $L^{p^{\prime}(x)}(\Omega)$ be the conjugate space of $L^{p(x)}(\Omega)$ with $\frac{1}{p(x)}+\frac{1}{p^{\prime}(x)}=1$. Then the following Höldertype inequality

$$
\left|\int_{\Omega} u v d x\right| \leq\left(\frac{1}{p^{-}}+\frac{1}{p^{\prime-}}\right)|u|_{p(x)}|v|_{p^{\prime}(x)}, \quad u \in L^{p(x)}(\Omega), v \in L^{p^{\prime}(x)}(\Omega)
$$

holds true.

The modular on the space $L^{p(x)}(\Omega)$ is the map $\rho_{p(x)}: L^{p(x)}(\Omega) \rightarrow \mathbb{R}$ defined by

$$
\rho_{p(x)}(u):=\int_{\Omega}|u|^{p(x)} d x
$$

Proposition 2.1. For all $u, v \in L^{p(x)}(\Omega)$, we have

1. $|u|_{p(x)}<1$ (resp. $\left.=1,>1\right) \Leftrightarrow \rho_{p(x)}(u)<1($ resp. $=1,>1)$.

2. $\min \left(|u|_{p(x)}^{p^{-}},|u|_{p(x)}^{p^{+}}\right) \leq \rho_{p(x)}(u) \leq \max \left(|u|_{p(x)}^{p^{-}},|u|_{p(x)}^{p^{+}}\right)$.

3. $\rho_{p(x)}(u-v) \rightarrow 0 \Leftrightarrow|u-v|_{p(x)} \rightarrow 0$.

Another property interesting the variable exponent Lebesgue space $L^{p(x)}(\Omega)$ is

Proposition 2.2. Let $p$ and $q$ be a measurable functions such that $p \in L^{\infty}(\Omega)$ and $1 \leq p(x) q(x) \leq \infty$, for a.e. $x \in \Omega$. Let $u \in L^{q(x)}(\Omega), u \neq 0$. Then

$$
\min \left(|u|_{p(x) q(x)}^{p^{+}},|u|_{p(x) q(x)}^{p^{-}}\right) \leq\left.\left.|| u\right|^{p(x)}\right|_{q(x)} \leq \max \left(|u|_{p(x) q(x)}^{p^{-}},|u|_{p(x) q(x)}^{p^{+}}\right) .
$$

For any positive integer $k$, let

$$
W^{k, p(x)}(\Omega)=\left\{u \in L^{p(x)}(\Omega): D^{\alpha} u \in L^{p(x)}(\Omega),|\alpha| \leq k\right\},
$$


where $\alpha=\left(\alpha_{1}, \alpha_{2}, \ldots, \alpha_{N}\right)$ is a multi-index, $|\alpha|=\sum_{i=1}^{N} \alpha_{i}$ and $D^{\alpha} u=\frac{\partial^{|\alpha|} u}{\partial^{\alpha} x_{1} \ldots . . \partial^{\alpha} N x_{n}}$. Then $W^{k, p(x)}(\Omega)$ is a separable and reflexive Banach space equipped with the norm

$$
\|u\|_{k, p(x)}=\sum_{|\alpha| \leq k}\left|D^{\alpha} u\right|_{p(x)}
$$

\section{Main results and proofs}

Throughout this paper, we consider that the problem (1.1) will be analyzed in the Orlicz-Sobolev space $W_{0}^{1} L_{\Phi}(\Omega)$.

Note that an eigenvalue for problem (1.1) satisfy the following definition.

Definition 3.1. A function $u \in W_{0}^{1} L_{\phi}(\Omega)$ is said to be a weak solution of problem (1.1) if $u \geq 0$ a.e. in $\Omega$ and it holds that

$$
\begin{aligned}
& \int_{\Omega}(a(|\nabla u(x)|) \nabla u(x) \nabla v(x)+a(|u(x)|) u(x) v(x)) d x-\lambda \int_{\Omega} V(x)|u(x)|^{m(x)-2} u(x) v(x) d x \\
+ & \mu \int_{\Omega} g(x, u(x)) v(x) d x=0, \quad \text { for all } v \in W_{0}^{1} L_{\Phi}(\Omega) .
\end{aligned}
$$

Let $E$ denote the Orlicz-Sobolev space $W_{0}^{1} L_{\phi}(\Omega)$. We define the energy functional $J_{\lambda, \mu}: E \rightarrow \mathbb{R}$ by

$$
J_{\lambda, \mu}(u)=\int_{\Omega}(\Phi(|\nabla u(x)|)+\Phi(|u(x)|)) d x-\lambda \int_{\Omega} \frac{V(x)}{m(x)}|u(x)|^{m(x)} d x+\mu \int_{\Omega} G(x, u(x)) d x, \forall u \in E .
$$

Standard arguments imply that $J_{\lambda, \mu}$ is well-defined on $E, J_{\lambda, \mu} \in C^{1}(E, \mathbb{R})$ and

$$
\begin{aligned}
<J_{\lambda, \mu}^{\prime}(u), v> & =\int_{\Omega}(a(|\nabla u(x)|) \nabla u(x) \nabla v(x)+a(|u(x)|) u(x) v(x)) d x \\
& -\lambda \int_{\Omega} V(x)|u(x)|^{m(x)-2} u(x) v(x) d x+\mu \int_{\Omega} g(x, u(x)) v(x) d x
\end{aligned}
$$

for all $u, v \in E$. Thus, we remark that the weak solution of (1.1) are exactly the critical points of the energy functional $J_{\lambda, \mu}$.

Proposition 3.1. (see [10,4]) Let the operator

$$
<\Lambda^{\prime}(u), v>=\int_{\Omega}(a(|\nabla u(x)|) \nabla u(x) \nabla v(x)+a(|u(x)|) u(x) v(x)) d x,
$$

defined from $W_{0}^{1} L_{\phi}(\Omega)$ to its dual space $\left(W_{0}^{1} L_{\phi}(\Omega)\right)^{*}$, is of type $\left(S_{+}\right)$, that is, if $u_{n} \rightarrow u$ and $\lim _{n \rightarrow+\infty} \sup _{n \rightarrow}<$ $\Lambda^{\prime}\left(u_{n}\right)\left(u_{n}-u\right)>\leq 0$, then $u_{n} \rightarrow u$ in $W_{0}^{1} L_{\phi}(\Omega)$.

In the following, we assume that the assumptions $\left(A_{1}\right)$ and $\left(A_{2}\right)$ holds. Then, the first main result of this work is given by the following Theorem. 
Theorem 3.2. Assume that the functions $m, q \in C(\bar{\Omega})$ verify the hypotheses

$$
m^{-}>\max \left(\varphi^{0}, q^{+}\right) \text {and } \max \left(r^{\prime}(x) m(x), q(x)\right)<\varphi_{0}^{*}=\frac{N \varphi_{0}}{N-\varphi_{0}} .
$$

Then, for any $\lambda, \mu>0$ the problem (1.1) has infinitely many weak solutions.

Denote by $r^{\prime}(x)$ the conjugate exponent of the function $r(x)$ where $r^{\prime}(x)=\frac{r(x)}{r(x)-1}$ and put $\alpha(x):=$ $\frac{r(x) m(x)}{r(x)-m(x)}$. From condition (3.1), we have $r^{\prime}(x) m(x)<\varphi_{0}^{*}(x), \forall x \in \bar{\Omega}, \quad \alpha(x)<\varphi_{0}^{*}(x), \forall x \in \bar{\Omega}$. Thus by Remark 2, the embeddings $E \hookrightarrow L^{r^{\prime}(x) m(x)}(\Omega)$ and $E \hookrightarrow L^{\alpha(x)}(\Omega)$ are compact and continuous.

The proof of Theorem 3.2 is based on a $\mathbb{Z}_{2}$-symmetric version for even functionals of the Mountain pass Theorem (see Theorem 9.12 in [27]).

Mountain pass Theorem. Let $\mathrm{X}$ be an infinite dimensional real Banach space and let $I \in C^{1}(X, R)$ be even, satisfying the Palais-Smale condition (i.e. any sequence $\left\{x_{n}\right\} \subset X$ such that $\left\{I\left(x_{n}\right)\right\}$ is bounded and $d I\left(x_{n}\right) \rightarrow 0$ in $X^{*}$ has a convergent subsequence) and $I(0)=0$. Suppose that

$\left(I_{1}\right)$ There exists two constants $\varrho, a$ such that $I(x) \geq \varrho>0$ if $\|x\|=a$.

$\left(I_{1}\right)$ For each finite dimensional subspace $X_{1} \subset X$, the set $\left\{x \in X_{1} ; I(x) \geq 0\right\}$ is bounded.

Then $I$ has an unbounded sequence of critical values.

We begin by proving there auxiliary Lemmas.

Lemma 3.3. Assume that the hypotheses of Theorem 3.2 are satisfied. Then, for any $\lambda, \mu>0$ there exists $\varrho, a>0$ such that $J_{\lambda, \mu} \geq a>0$ for any $u \in E$ with $\|u\|=\varrho$.

Proof. Since the embedding $E \hookrightarrow L^{r^{\prime}(x) m(x)}(\Omega)$ is continuous, we can find a constant $N_{1}>0$ such that

$$
|u|_{r^{\prime}(x) m(x)} \leq N_{1}\|u\|, \quad \forall u \in E .
$$

Let us fix $\varrho \in(0,1)$ such that $\varrho<\frac{1}{N_{1}}$. Then relation (3.2) implies $|u|_{r^{\prime}(x) m(x)}<1$, for all $u \in E$ with $\|u\|=\varrho$. Thus, by Hölder inequality (2.5) and Proposition 2.2, we have

$$
\int_{\Omega} V(x)|u|^{m(x)} d x \leq\left.\left.|V|_{r(x)}|| u\right|^{m(x)}\right|_{r^{\prime}(x)} \leq|V|_{r(x)}|u|_{r^{\prime}(x) m(x)}^{m^{-}},
$$

for all $u \in E$ with $\|u\|=\varrho$.

Combining (3.2) and (3.3), we obtain

$$
\int_{\Omega} V(x)|u|^{m(x)} d x \leq N_{1}^{m^{-}}|V|_{r(x)}\|u\|^{m^{-}},
$$

for all $u \in E$ with $\|u\|=\varrho$.

On the other hand, using Lemma 2.1, for all $u \in E$ with $\|u\|=\varrho$. We have

$$
\int_{\Omega}(\Phi(|\nabla u|)+\Phi(|u|)) d x \geq\|u\|^{\varphi^{0}} .
$$


Hence, from relations (3.4) and (3.5), we deduce that, for any $u \in E$ with $\|u\|=\varrho$, the following inequalities hold true

$$
\begin{aligned}
J_{\lambda, \mu}(u) & =\int_{\Omega}(\Phi(|\nabla u(x)|)+\Phi(|u(x)|)) d x-\lambda \int_{\Omega} \frac{1}{m(x)} V(x)|u(x)|^{m(x)} d x+\mu \int_{\Omega} G(x, u(x)) d x \\
& \geq \int_{\Omega}(\Phi(|\nabla u(x)|)+\Phi(|u(x)|)) d x-\frac{\lambda}{m^{-}} \int_{\Omega} V(x)|u(x)|^{m(x)} d x+\mu \int_{\Omega} G(x, u(x)) d x \\
& \geq \int_{\Omega}(\Phi(|\nabla u(x)|)+\Phi(|u(x)|)) d x-\frac{\lambda}{m^{-}} \int_{\Omega} V(x)|u(x)|^{m(x)} d x \\
& \geq\|u\|^{\varphi^{0}}-\frac{\lambda}{m^{-}}|V(x)|_{r(x)}|u|_{r(x)^{\prime} m(x)}^{m^{-}} \\
& \geq\|u\|^{\varphi^{0}}-\frac{\lambda}{m^{-}}|V(x)|_{r(x)} N_{1}^{m^{-}}\|u\|^{m^{-}} .
\end{aligned}
$$

Let $K_{\lambda}(t)=t^{\varphi^{0}}-\frac{\lambda}{m^{-}}|V(x)|_{r(x)} N_{1}^{m^{-}} t^{m^{-}}, t>0$. It is easy to see that $K_{\lambda}(t)>0$ for all $t \in\left(0, t_{1}\right)$, where $t_{1}<\left(\frac{m^{-}}{\lambda N_{1}^{m^{-}}|V(x)|_{r(x)}}\right)^{\frac{1}{m^{-}-\varphi^{0}}}$.

So for any $\lambda, \mu>0$ we can chooser $\varrho, a>0$ such that $J_{\lambda, \mu} \geq a>0$ for all $u \in E$ with $\|u\|=\varrho$. This completes the proof of Lemma.

Lemma 3.4. Assume that the hypotheses of Theorem 3.2 are verified and let $E_{1}$ be a finite dimensional subspace of $E$. Then the set $S=\left\{u \in E_{1} ; J_{\lambda, \mu}(u) \geq 0\right\}$ is bounded in $E$.

Proof. Using Lemma (2.1), we have

$$
\int_{\Omega}(\Phi(|\nabla u(x)|)+\Phi(|u(x)|)) d x \leq K_{1}\left(\|u\|^{\varphi_{0}}+\|u\|^{\varphi^{0}}\right) \forall u \in E,
$$

where $K_{1}$ is positive constant. On the other hand, since $E$ is continuously embedded in $L^{q(x)}(\Omega)$, it follows that there exists a positive constant $N_{2}>0$ such that

$$
|u(x)|_{q(x)} \leq N_{2}\|u\|, \quad \forall u \in E .
$$

In fact, assumption $\left(A_{1}\right)$ implies

$$
\int_{\Omega} G(x, u) d x \leq C_{2} \int_{\Omega}|u|^{q(x)} d x \leq C_{2}\left(|u(x)|_{q(x)}^{q^{-}}+|u(x)|_{q(x)}^{q^{+}}\right), \quad \forall u \in E .
$$

The last two inequalities show that there exists a positive constant $K_{2}(\mu)$ such that

$$
\mu \int_{\Omega} G(x, t) d x \leq \mu C_{2}\left(N_{2}^{q^{-}}\|u\|^{q^{-}}+N_{2}^{q^{+}}\|u\|^{q^{+}}\right) \leq K_{2}(\mu)\left(\|u\|^{q^{-}}+\|u\|^{q^{+}}\right), \quad \forall u \in E .
$$

By inequalities (3.6) and (3.9) we get

$$
J_{\lambda, \mu}(u) \leq K_{1}\left(\|u\|^{\varphi_{0}}+\|u\|^{\varphi^{0}}\right)+K_{2}(\mu)\left(\|u\|^{q^{-}}+\|u\|^{q^{+}}\right)-\frac{\lambda}{m^{+}} \int_{\Omega} V(x)|u(x)|^{m(x)} d x
$$

for all $u \in E$. 
Let $u \in E$ be arbitrary but fixed. We define

$$
\Omega_{<}=\{x \in \Omega,|u(x)|<1\} . \Omega_{\geq}=\Omega \backslash \Omega_{<} .
$$

Then we have

$$
\begin{aligned}
J_{\lambda, \mu}(u) & =K_{1}\left(\|u\|^{\varphi_{0}}+\|u\|^{\varphi^{0}}\right)+K_{2}(\mu)\left(\|u\|^{q^{-}}+\|u\|^{q^{+}}\right)-\frac{\lambda}{m^{+}} \int_{\Omega} V(x)|u(x)|^{m(x)} d x \\
& \leq K_{1}\left(\|u\|^{\varphi_{0}}+\|u\|^{\varphi^{0}}\right)+K_{2}(\mu)\left(\|u\|^{q^{-}}+\|u\|^{q^{+}}\right)-\frac{\lambda}{m^{+}} \int_{\Omega_{\geq}} V(x)|u(x)|^{m(x)} d x \\
& \leq K_{1}\left(\|u\|^{\varphi_{0}}+\|u\|^{\varphi^{0}}\right)+K_{2}(\mu)\left(\|u\|^{q^{-}}+\|u\|^{q^{+}}\right)-\frac{\lambda}{m^{+}} \int_{\Omega_{\geq}} V(x)|u(x)|^{m^{-}} d x \\
& \leq K_{1}\left(\|u\|^{\varphi_{0}}+\|u\|^{\varphi^{0}}\right)+K_{2}(\mu)\left(\|u\|^{q^{-}}+\|u\|^{q^{+}}\right)-\frac{\lambda}{m^{+}} \int_{\Omega} V(x)|u(x)|^{m^{-}} d x \\
& +\frac{\lambda}{m^{+}} \int_{\Omega_{<}} V(x)|u(x)|^{m^{-}} d x .
\end{aligned}
$$

But for each $\lambda>0$ there exists positive constant $K_{3}(\lambda)$ such that

$$
\frac{\lambda}{m^{+}} \int_{\Omega_{<}} V(x)|u(x)|^{m^{-}} d x \leq K_{3}(\lambda), \quad \forall u \in E .
$$

We have

$$
-\frac{\lambda}{m^{+}} \int_{\Omega} V(x)|u(x)|^{m^{-}} d x \leq-\frac{\lambda}{m^{+}}|V|_{r(x)}|u|_{r^{\prime}(x) m(x)}^{m^{-}}, \quad \forall u \in E .
$$

In the finite dimensional $E_{1}$ the norm $|u|_{r^{\prime}(x) m(x)}$ and $\|$.$\| are equivalent, so there exists a positive$ constant $K=K\left(E_{1}\right)$

$$
\|u\| \leq K\left(E_{1}\right)|u|_{r^{\prime}(x) m(x)} .
$$

So, there exists a positive constant $K_{4}(\lambda)$ such that

$$
J_{\lambda, \mu}(u) \leq K_{1}\left(\|u\|^{\varphi_{0}}+\|u\|^{\varphi^{0}}\right)+K_{2}(\mu)\left(\|u\|^{q^{-}}+\|u\|^{q^{+}}\right)+K_{3}(\lambda)-K_{4}(\lambda)\|u\|^{m^{-}}, \quad \forall u \in E_{1} .
$$

Hence

$$
K_{1}\left(\|u\|^{\varphi_{0}}+\|u\|^{\varphi^{0}}\right)+K_{2}(\mu)\left(\|u\|^{q^{-}}+\|u\|^{q^{+}}\right)+K_{3}(\lambda)-K_{4}(\lambda)\|u\|^{m^{-}} \geq 0, \quad \forall u \in S .
$$

And since $m^{-}>\max \left(\varphi^{0}, q^{+}\right)$, we conclude that $S$ is bounded in $E$.

Lemma 3.5. If $\left\{u_{n}\right\} \in E$ is a sequence which satisfies the properties

$$
\begin{aligned}
& \left|J_{\lambda, \mu}\left(u_{n}\right)\right|<C_{3}, \\
& d J_{\lambda, \mu}\left(u_{n}\right) \rightarrow 0 \text { as } n \rightarrow \infty,
\end{aligned}
$$

where $C_{3}$ is a positive constant, then $\left\{u_{n}\right\}$ possesses a convergent subsequence. 
Proof. First we show that $\left\{u_{n}\right\}$ is bounded in $E$. If not,we may assume that $\left\|u_{n}\right\| \rightarrow \infty$ as $n \rightarrow \infty$. Thus we may consider that $\left\|u_{n}\right\|>1$ for any integer $n$. Using (3.12) it follows that there exists $N^{\prime}>0$ such that for any $n>N^{\prime}$ we have

$$
\left\|d J_{\lambda, \mu}\left(u_{n}\right)\right\| \leq 1 \text {. }
$$

On the other hand, for all $n>N^{\prime}$ fixed, the application $E \ni v \rightarrow\left\langle d J_{\lambda, \mu}\left(u_{n}\right), v>\right.$ is linear and continuous. The above information implies that

$$
\left|<d J_{\lambda, \mu}\left(u_{n}\right), v>\right| \leq\left\|d J_{\lambda, \mu}\left(u_{n}\right)\right\|\|v\| \leq\|v\|, \quad \forall v \in E \quad n>N^{\prime} .
$$

Setting $v=u_{n}$ we have

$$
-\left\|u_{n}\right\| \leq \int_{\Omega}\left(\Phi\left(\left|\nabla u_{n}\right|\right)+\Phi\left(\left|u_{n}\right|\right)\right) d x-\lambda \int_{\Omega} \frac{1}{m(x)} V(x)\left|u_{n}\right|^{m(x)} d x+\mu \int_{\Omega} G\left(x, u_{n}\right) d x \leq\left\|u_{n}\right\|,
$$

for all $n>N^{\prime}$. We obtain

$$
-\left\|u_{n}\right\|-\int_{\Omega}\left(\Phi\left(\left|\nabla u_{n}\right|\right)+\Phi\left(\left|u_{n}\right|\right)\right) d x-\mu \int_{\Omega} G\left(x, u_{n}\right) d x \leq-\lambda \int_{\Omega} \frac{1}{m(x)} V(x)\left|u_{n}\right|^{m(x)} d x,
$$

for all $n>N^{\prime}$. Provided that $\left\|u_{n}\right\|>1$ relation (3.11) and (3.13) imply

$$
\begin{aligned}
C_{3}>J_{\lambda, \mu}\left(u_{n}\right) & \geq\left(1-\frac{1}{m^{-}}\right) \int_{\Omega}\left(\Phi\left(\left|\nabla u_{n}\right|\right)+\Phi\left(\left|u_{n}\right|\right)\right) d x+\mu\left(1-\frac{1}{m^{-}}\right) \int_{\Omega} G(x, u(x)) d x-\frac{1}{m^{-}}\left\|u_{n}\right\| \\
& \geq\left(1-\frac{1}{m^{-}}\right) \int_{\Omega}\left(\Phi\left(\left|\nabla u_{n}\right|\right)+\Phi\left(\left|u_{n}\right|\right)\right) d x-\frac{1}{m^{-}}\left\|u_{n}\right\| \\
& \geq\left(1-\frac{1}{m^{-}}\right)\left\|u_{n}\right\|^{\varphi_{0}}-\frac{1}{m^{-}}\left\|u_{n}\right\|,
\end{aligned}
$$

letting $n \rightarrow \infty$ we obtain a contradiction. It follows that $\left\{u_{n}\right\}$ is bounded in $E$. And we deduce that there exists a subsequence, again denoted by $\left\{u_{n}\right\}$, and $u \in E$ such that $\left\{u_{n}\right\}$ converges weakly to $u$ in $E$. Since $E$ is compactly embedded in $L^{\alpha(x)}(\Omega)$ and $L^{q(x)}(\Omega)$ then $\left\{u_{n}\right\}$ converges strongly to $u$ in $L^{\alpha(x)}(\Omega)$ and $L^{q(x)}(\Omega)$, respectively. For the strong convergence of $\left\{u_{n}\right\}$ in $E$, we need the following Proposition.

\section{Proposition 3.2.}

1. $\lim _{n \rightarrow \infty} \int_{\Omega} V(x)\left|u_{n}\right|^{m(x)-2} u_{n}\left(u_{n}-u\right) d x=0$.

2. $\lim _{n \rightarrow \infty} \int_{\Omega} g\left(x, u_{n}\right)\left(u_{n}-u\right) d x=0$.

Proof. Let us start firstly by the proof of assertion 1. For this fact, we use the Hölder inequality (2.5) and we have the following

$$
\begin{aligned}
\left.\int_{\Omega}|V(x)| u_{n}\right|^{m(x)-2} u_{n}\left(u_{n}-u\right) \mid d x & \leq\left.\left.|V|_{r(x)}|| u_{n}\right|^{m(x)-2} u_{n}\left(u_{n}-u\right)\right|_{r^{\prime}(x)} \\
& \leq\left.\left.|V|_{r(x)}|| u_{n}\right|^{m(x)-2} u_{n}\right|_{\frac{m(x)}{m(x)-1}}\left|u_{n}-u\right|_{\alpha(x)} .
\end{aligned}
$$


Moreover, using Proposition 2.2 in the case where $\left.\left.|| u_{n}\right|^{m(x)-2} u_{n}\right|_{\frac{m(x)}{m(x)-1}}>1$, we deduce that

$$
\left.\left.|| u_{n}\right|^{m(x)-2} u_{n}\right|_{\frac{m(x)}{m(x)-1}} \leq\left|u_{n}\right|_{m(x)}^{m^{+}} .
$$

Finally The compact embedding $X \hookrightarrow L^{m(x)}(\Omega)$ ends the proof.

For the proof of the second assertion, similar arguments showed that

$$
\int_{\Omega}\left|g\left(x, u_{n}\right)\left(u_{n}-u\right)\right| d x \leq C_{0} \int_{\Omega}\left|u_{n}\right|^{q(x)-2} u_{n}\left(u_{n}-u\right) d x \leq\left.\left. C_{0}|| u_{n}\right|^{q(x)-2} u_{n}\right|_{\frac{q(x)}{q(x)-1}}\left|u_{n}-u\right|_{q(x)},
$$

then if

$$
\left.\left.|| u_{n}\right|^{q(x)-2} u_{n}\right|_{\frac{q(x)}{q(x)-1}}>1,
$$

by Proposition 2.2, there exists $C>0$ such that

$$
\left.\left.|| u_{n}\right|^{q(x)-2} u_{n}\right|_{\frac{q(x)}{q(x)-1}}<\left|u_{n}\right|_{q(x)}^{C},
$$

and this ends the proof.

Since $d J_{\lambda, \mu}\left(u_{n}\right) \rightarrow 0$ and $u_{n}$ is bounded in $E$, one has

$$
\begin{aligned}
\left|\left\langle d J_{\lambda, \mu}\left(u_{n}\right), u_{n}-u\right\rangle\right| & \leq\left|\left\langle d J_{\lambda, \mu}\left(u_{n}\right), u_{n}\right\rangle\right|+\left|\left\langle d J_{\lambda, \mu}\left(u_{n}\right), u\right\rangle\right| \\
& \leq\left\|d J_{\lambda, \mu}\left(u_{n}\right)\right\|\left\|u_{n}\right\|+\left\|d J_{\lambda, \mu}\left(u_{n}\right)\right\|\|u\| .
\end{aligned}
$$

So

$$
\lim _{n \rightarrow \infty}\left\langle d J_{\lambda, \mu}\left(u_{n}\right), u_{n}-u\right\rangle=0 .
$$

Moreover, using Proposition 3.2 and the last relations we deduce that

$$
\lim _{n \rightarrow \infty} \int_{\Omega}\left(a\left(\left|\nabla u_{n}\right|\right) \nabla u_{n}\left(\nabla u_{n}-\nabla u\right)+a\left(\left|u_{n}\right|\right) u_{n}\left(\nabla u_{n}-\nabla u\right)\right) d x=0 .
$$

Now, Proposition 3.1, ensures that $\left\{u_{n}\right\}$ converges strongly to $u$ in $E$. The proof of Lemma 3.5 is complete.

Proof of Theorem 3.2. It is clear that the functional $J_{\lambda, \mu}$ is even and verifies $J_{\lambda, \mu}(0)=0$. Lemma 3.3, Lemma 3.4 and Lemma 3.5 implies that the Mountain Pass Theorem can be applied to the functional $J_{\lambda, \mu}$. We conclude that problem (1.1) has infinitely many weak solutions in $E$. The proof of Theorem 3.2 is complete.

The second main Theorem for problem (1.1) can be described as follows.

Theorem 3.6. For $\mu>0$, suppose that the functions $m, q \in C(\bar{\Omega})$ satisfy the conditions

$$
m^{-}<\min \left(\varphi_{0}, q^{-}\right) \text {and } \max \left(r^{\prime}(x) m(x), q(x)\right)<\varphi_{0}^{*}=\frac{N \varphi_{0}}{N-\varphi_{0}} .
$$

Then, there exists $\lambda^{*}$ such that for any $\lambda \in\left(0, \lambda^{*}\right)$ problem (1.1) has a nontrivial weak solution in $W_{0}^{1} L_{\phi}(\Omega)$. 
The key argument in the proof of this Theorem is related to Ekeland's variational principle. In order to apply it we need the following Lemmas.

Lemma 3.7. Assume that the hypotheses of Theorem 3.6 are satisfied. For all $\mu>0$ and all $\rho \in(0,1)$ there exist $\lambda^{*}>0$ and $b>0$ such that, for all $u \in E$ with $\|u\|=\rho$,

$$
J_{\lambda, \mu} \geq b>0 \text { for any } \lambda \in\left(0, \lambda^{*}\right) .
$$

Proof. Since the embedding $E \hookrightarrow L^{r^{\prime}(x) m(x)}(\Omega)$ is continuous. Then, there exists a positive constant $N_{3}$ such that

$$
|u|_{r^{\prime}(x) m(x)} \leq N_{3}\|u\|, \text { for all } u \in E .
$$

We fix $\rho<(0,1)$ such that $\rho<\min \left(1,1 / N_{3}\right)$. Then for all $u \in E$ with $\|u\|=\rho$ we deduce that

$$
|u|_{r^{\prime}(x) m(x)}<1 \text {. }
$$

Using Lemma 2.1 Proposition 2.2, Hölder inequality (2.5) and relation (3.15), we deduce that for any $u \in E$ with $\|u\|=\rho$ the following inequalities hold true

$$
\begin{aligned}
J_{\lambda, \mu}(u) & =\int_{\Omega}(\Phi(|\nabla u(x)|)+\Phi(|u(x)|)) d x-\lambda \int_{\Omega} \frac{1}{m(x)} V(x)|u(x)|^{m(x)} d x+\mu \int_{\Omega} G(x, u(x)) d x \\
& \geq \int_{\Omega}(\Phi(|\nabla u(x)|)+\Phi(|u(x)|)) d x-\frac{\lambda}{m^{-}} \int_{\Omega} V(x)|u(x)|^{m(x)} d x+\mu \int_{\Omega} G(x, u(x)) d x \\
& \geq\|u\|^{\varphi^{0}}-\frac{\lambda}{m^{-}}|V(x)|_{r(x)}|u(x)|_{r(x)^{\prime} m(x)}^{m^{-}} \\
& \geq\|u\|^{\varphi^{0}}-\frac{\lambda}{m^{-}}|V(x)|_{r(x)} N_{3}^{m^{-}}\|u(x)\|^{m^{-}} \\
& \geq \rho^{m^{-}}\left(\rho^{\varphi^{0}-m^{-}}-\frac{\lambda}{m^{-}}|V(x)|_{r(x)} N_{3}^{m^{-}}\right) .
\end{aligned}
$$

By the above inequality, we remark that if we define

$$
\lambda^{*}=\frac{m^{-}}{2 N_{3}^{m^{-}}|V(x)|_{r(x)}} \rho^{\varphi^{0}-m^{-}}
$$

then, for any $\lambda \in\left(0, \lambda^{*}\right)$, there exists $b=\frac{\rho^{\varphi^{0}}}{2}>0$ such that

$$
J_{\lambda, \mu} \geq b>0, \forall \mu>0, \forall u \in E \text { with }\|u\|=\rho .
$$

The proof of Lemma 3.7 is complete.

Lemma 3.8. Suppose that the conditions of Theorem 3.6 are verified. Then there is $\psi \in E$ such that $\psi \geq 0, \psi \neq 0$ and $J_{\lambda, \mu}(t \psi)<0$, for $t>0$ small enough.

Proof. Let $l=\min \left(\varphi_{0}, q^{-}\right)$. Since $m^{-}<l$, then let $\epsilon_{0}>0$ be such that $m^{-}+\epsilon_{0}<l$. In the same time, the fact that $m \in C(\bar{\Omega})$ yields the existence of an open nonempty set $\Omega_{0} \subset \subset \Omega$ so that $\left|m(x)-m^{-}\right|<\epsilon_{0}$ for all $x \in \Omega_{0}$. Thus, we conclude that $m(x) \leq m^{-}+\epsilon_{0}<l$ for all $x \in \overline{\Omega_{0}}$. 
On the other hand, let $\psi \in C_{0}^{\infty}(\Omega)$ be such that $\operatorname{supp}(\psi) \supset \overline{\Omega_{0}}, \psi(x)=1$ for all $x \in \overline{\Omega_{0}}$ and $0 \leq \psi \leq 1$ in $\Omega$. Then using the above information for any $t \in(0,1)$ we have

$$
\begin{aligned}
J_{\lambda, \mu}(t \psi) & =\int_{\Omega}(\Phi(|\nabla(t \psi)|)+\Phi(|t \psi|)) d x-\lambda \int_{\Omega} \frac{1}{m(x)} V(x)|t \psi|^{m(x)} d x+\mu \int_{\Omega} G(x, t \psi) d x \\
& \leq t^{\varphi_{0}} \int_{\Omega}(\Phi(|\nabla \psi|)+\Phi(|\psi|)) d x+\mu t^{q^{-}} \int_{\Omega} G(x, \psi) d x-\frac{\lambda}{m^{+}} t^{m^{-}+\epsilon_{0}} \int_{\Omega_{0}} V(x)|\psi|^{m(x)} d x \\
& \leq t^{l}\left[\int_{\Omega}(\Phi(|\nabla \psi|)+\Phi(|\psi|)) d x+\mu \int_{\Omega} G(x, \psi) d x\right]-\frac{\lambda}{m^{+}} t^{m^{-}+\epsilon_{0}} \int_{\Omega_{0}} V(x)|\psi|^{m(x)} d x \\
& \leq t^{l}\left[\max \left(\|\psi\|^{\varphi_{0}},\|\psi\|^{\varphi^{0}}\right)+\mu \int_{\Omega} G(x, \psi) d x\right]-\frac{\lambda}{m^{+}} t^{m^{-}+\epsilon_{0}} \int_{\Omega_{0}} V(x)|\psi|^{m(x)} d x
\end{aligned}
$$

Therefore

$$
J_{\lambda, \mu}(t \psi)<0 \text { for } t<\delta^{\frac{1}{l-m^{-}-\epsilon_{0}}},
$$

with

$$
0<\delta<\min \left\{1, \frac{\lambda \int_{\Omega_{0}} V(x)|\psi|^{m(x)} d x}{m^{+}\left[\max \left(\|\psi\| \varphi^{\varphi_{0}},\|\psi\|^{\varphi^{0}}\right)+\mu \int_{\Omega} G(x, \psi) d x\right]}\right\} .
$$

Finally, we point out that $\max \left(\|\psi\|^{\varphi_{0}},\|\psi\|^{\varphi^{0}}\right)+\mu \int_{\Omega} G(x, \psi) d x>0$. In fact if $\max \left(\|\psi\| \varphi^{\varphi_{0}},\|\psi\|^{\varphi^{0}}\right)+$ $\mu \int_{\Omega} G(x, \psi) d x=0$, one has $\|\psi\|=0$ then $\psi=0$ in $\Omega$ which is a contradiction. The proof of Lemma 3.8 is complete.

We split the proof of Theorem 3.6 into two steps.

Proof of Theorem 3.6. Step 1. Let $\lambda^{*}>0$ be defined as in (3.16) and $\lambda \in\left(0, \lambda^{*}\right)$. By Lemma 3.7 it follows that on the boundary of the ball centered at the origin and of radius $\rho$ in $E$, denoted by $B_{\rho}(0)$, we have

$$
\inf _{\partial B_{\rho}(0)} J_{\lambda, \mu}>0
$$

At the same time, by Lemma 3.8, there exists $\psi \in E$ such that $J_{\lambda, \mu}(t \psi)<0$ for all $t>0$ small enough. Moreover, using Hölder inequality (2.5), Proposition 2.2, Lemma 2.1 and relation (3.15), we deduce that for any $u \in B_{\rho}(0)$ we have

$$
J_{\lambda, \mu}(u) \geq\|u\|^{\varphi^{0}}-\frac{\lambda}{m^{-}}|V(x)|_{r(x)} N_{3}^{m^{-}}\|u(x)\|^{m^{-}} .
$$

It follows that

$$
-\infty<\underline{c}:=\frac{\inf }{B_{\rho}(0)} J_{\lambda, \mu}<0 .
$$


Now, we let $0<\epsilon<\inf _{\partial B_{\rho}(0)} J_{\lambda, \mu}-\inf _{B_{\rho}(0)} J_{\lambda, \mu}$. Using the above information, the functional $J_{\lambda, \mu}$ : $\overline{B_{\rho}(0)} \longrightarrow \mathbb{R}$, is lower bounded on $\overline{B_{\rho}(0)}$ and $J_{\lambda, \mu} \in C^{1}\left(\overline{B_{\rho}(0)}, \mathbb{R}\right)$. Then by Ekeland's variational principle, there exists $u_{\epsilon} \in \overline{B_{\rho}(0)}$ such that

$$
\left\{\begin{array}{l}
\underline{c} \leq J_{\lambda, \mu}\left(u_{\epsilon}\right) \leq \underline{c}+\epsilon, \\
0<J_{\lambda, \mu}(u)-J_{\lambda, \mu}\left(u_{\epsilon}\right)+\epsilon \cdot\left\|u-u_{\epsilon}\right\|, \quad u \neq u_{\epsilon} .
\end{array}\right.
$$

Then, we infer the following inequalities:

$$
J_{\lambda, \mu}\left(u_{\epsilon}\right) \leq \inf _{\overline{B_{\rho}}(0)} J_{\lambda, \mu}+\epsilon \leq \inf _{B_{\rho}(0)} J_{\lambda, \mu}+\epsilon<\inf _{\partial B_{\rho}(0)} J_{\lambda, \mu},
$$

meaning that $u_{\epsilon} \in B_{\rho}(0)$.

Now, we define $I_{\lambda, \mu}: \overline{B_{\rho}(0)} \longrightarrow \mathbb{R}$ by $I_{\lambda, \mu}(u)=J_{\lambda, \mu}(u)+\epsilon \cdot\left\|u-u_{\epsilon}\right\|$. It is clear that $u_{\epsilon}$ is a minimum point of $I_{\lambda, \mu}$ and thus

$$
\frac{I_{\lambda, \mu}\left(u_{\epsilon}+t \cdot v\right)-I_{\lambda, \mu}\left(u_{\epsilon}\right)}{t} \geq 0, \text { for small } t>0 \text { and every } v \in B_{1}(0) .
$$

This shows that

$$
\frac{J_{\lambda, \mu}\left(u_{\epsilon}+t \cdot v\right)-J_{\lambda, \mu}\left(u_{\epsilon}\right)}{t}+\epsilon \cdot\|v\| \geq 0 .
$$

When $t$ tends to $0^{+}$, we have that

$$
\left\langle d J_{\lambda, \mu}\left(u_{\epsilon}\right), v\right\rangle+\epsilon \cdot\|v\| \geq 0 .
$$

This gives

$$
\left\|d J_{\lambda, \mu}\left(u_{\epsilon}\right)\right\| \leq \epsilon
$$

Step 2. From (3.18), we deduce that there exists a sequence $\left\{w_{n}\right\} \subset B_{\rho}(0)$ such that

$$
J_{\lambda, \mu}\left(w_{n}\right) \longrightarrow \underline{c}<0 \quad \text { and } \quad d J_{\lambda, \mu}\left(w_{n}\right) \longrightarrow 0_{E^{*}} .
$$

It is clear that $\left\{w_{n}\right\}$ is bounded in $E$. Thus, there exists $w$ in $E$ such that, up to a subsequence, $\left\{w_{n}\right\}$ converges weakly to $w$ in $E$.

Since $\alpha(x), q(x)<\varphi_{0}^{*}(x)$ for all $x \in \bar{\Omega}$ we deduce that there exists a compact embedding $E \hookrightarrow$ $L^{\alpha(x)}(\Omega), E \hookrightarrow L^{q(x)}(\Omega)$ respectively and consequently $\left\{w_{n}\right\}$ converges strongly in $L^{\alpha(x)}(\Omega)$ and $L^{q(x)}(\Omega)$. Using similar arguments than those used in proof of Lemma 3.5 we deduce that $\left\{w_{n}\right\}$ converges strongly to $w$ in $E$.

Since $J_{\lambda, \mu} \in C^{1}(E, \mathbb{R})$, we conclude

$$
d J_{\lambda, \mu}\left(w_{n}\right) \rightarrow d J_{\lambda, \mu}(w) \text {, as } n \rightarrow \infty .
$$

Relations (3.19) and (3.20) showed that $d J_{\lambda, \mu}(w)=0$ and thus $w$ is a weak solution for problem (1.1). Moreover, by relation (3.19), it follows that $J_{\lambda, \mu}(w)<0$ and thus, $w$ is a nontrivial weak solution for (1.1).

Now, we present the last main result as follows. 
Theorem 3.9. We adopt the fact that the functions $m, q \in C(\bar{\Omega})$ fulfill the following hypotheses

$$
m^{+}<\varphi^{0} \text { and } r^{\prime}(x) m(x)<\varphi_{0}^{*}=\frac{N \varphi_{0}}{N-\varphi_{0}} .
$$

Then, for $\mu>0$, there is $\lambda^{* *}>0$ so that every $\lambda \in\left[\lambda^{* *}, \infty\right)$ is an eigenvalue of the problem (1.1).

Under the hypotheses of this Theorem, we want to construct a global minimizer of the functional $J_{\lambda, \mu}$ by the direct method in calculus of variations. For this, we start with the following auxiliary results.

Lemma 3.10. Assume that hypotheses of Theorem 3.9 are fulfilled. Then, for any $\lambda, \mu>0$, the functional $J_{\lambda, \mu}$ is coercive.

Proof. For each $u \in E$ with $\|u\|>1$ and $\lambda, \mu>0$, Lemma 2.1, Remark 2 imply

$$
\begin{aligned}
J_{\lambda, \mu}(u) & \geq\|u\|^{\varphi^{0}}-\frac{\lambda}{m^{-}} \int_{\Omega} V(x)|u(x)|^{m(x)} d x+\mu \int_{\Omega} G(x, u(x)) d x \\
& \geq\|u\|^{\varphi^{0}}-\frac{\lambda}{m^{-}}|V(x)|_{r(x)} \max \left(|u(x)|_{r(x)^{\prime} m(x)}^{m^{-}},|u(x)|_{r(x)^{\prime} m(x)}^{m^{+}}\right) \\
& \geq\|u\|^{\varphi^{0}}-\frac{\lambda}{m^{-}}|V(x)|_{r(x)} \max \left(N_{1}^{m^{-}}\|u(x)\|^{m^{-}}, N_{1}^{m^{+}}\|u(x)\|^{m^{+}}\right) .
\end{aligned}
$$

Since $m^{+}<\varphi^{0}$, the above inequality implies that $J_{\lambda, \mu} \rightarrow \infty$ as $\|u\| \rightarrow \infty$, that is, $J_{\lambda, \mu}$ is coercive. The proof of Lemma 3.10 is complete.

Lemma 3.11. The functional $\Lambda$ is weakly lower semi-continuous.

Proof. We have the functional $\Lambda: E \rightarrow \mathbb{R}$ defined by

$$
\Lambda(u(x))=\int_{\Omega}[\Phi(|\nabla u(x)|)+\Phi(|u(x)|)] d x, \quad \forall u \in E .
$$

We show that $\Lambda$ is weakly lower semi-continuous. For this purpose, we fix $u \in E$ and $\epsilon>0$. Since $\Lambda$ is convex (because $\Phi$ is convex) we deduce that for any $v \in E$ the following inequality hold true

$$
\Lambda(v) \geq \Lambda(u)+<\Lambda^{\prime}(u), v-u>
$$

or

$$
\begin{aligned}
\Lambda(v) & \geq \Lambda(u(x))-\int_{\Omega}(a(|\nabla u(x)|)|\nabla u(x)| \cdot|\nabla v(x)-\nabla u(x)|+a(|u(x)|)|u(x)||v(x)-u(x)|) d x \\
& =\Lambda(u(x))-\int_{\Omega}(\varphi(|\nabla u(x)|)|\nabla v(x)-\nabla u(x)|+\varphi(|u(x)|)|v(x)-u(x)|) d x
\end{aligned}
$$

Next, we claim that $\varphi(|u(x)|) \in L_{(\Phi) *}(\Omega)$ provided that $u \in L_{(\Phi)}(\Omega)$, where $(\Phi)^{*}$ is the conjugate Young function of $\Phi$. Thus by relation (2.1) we find

$$
\begin{aligned}
\Lambda(v) & \geq \Lambda(u(x))-2\left[|\varphi(|\nabla u(x)|)|_{(\Phi)^{*}}|\nabla v(x)-\nabla u(x)|_{\Phi}+|\varphi(|u(x)|)|_{\left.(\Phi)^{*} \cdot|v(x)-u(x)|_{\Phi}\right]}\right. \\
& \geq \Lambda(u(x))-C^{\prime}\|u(x)-v(x)\| \\
& \geq \Lambda(u(x))-\epsilon
\end{aligned}
$$


for all $v \in E$ with $\|u(x)-v(x)\|<\delta=\frac{\epsilon}{C^{\prime}}$, where $C^{\prime}$ is a positive constant. The proof of Lemma 3.11 is complete.

Proposition 3.3. The functional $J_{\lambda, \mu}$ is weakly lower semi continuous.

Proof. Using Lemma 3.11 we have that $\Lambda$ is weakly lower semi continuous. we show that $J_{\lambda, \mu}$ is weakly lower semi continuous. Let $u_{n} \subset E$ be a sequence which converges weak by to $u$ in $E$. By Lemma (3.11) we deduce

$$
\Lambda(u) \leq \liminf _{n \rightarrow \infty} \Lambda\left(u_{n}\right)
$$

On the other hand

$$
\liminf _{n \rightarrow \infty} \int_{\Omega} V(x)\left|u_{n}\right|^{m(x)} d x=\int_{\Omega} V(x)|u|^{m(x)} d x,
$$

and

$$
\liminf _{n \rightarrow \infty} \int_{\Omega} G\left(x, u_{n}\right) d x=\int_{\Omega} G(x, u) d x,
$$

Thus, we find

$$
J_{\lambda, \mu}(u) \leq \liminf _{n \rightarrow \infty} J_{\lambda, \mu}\left(u_{n}\right) .
$$

Therefore, $J_{\lambda, \mu}$ is weakly lower semi continuous and Proposition 3.3 is proved.

Proof of Theorem 3.9. Using Lemma 3.10 and Proposition 3.3, we infer that $J_{\lambda, \mu}$ is coercive and weakly lower semi continuous in $E$, for all $\lambda, \mu>0$. Then Theorem 1.2 in [22] implies that there exists $\underline{u} \in E$ a global minimizer of problem (1.1).

We intend to show that $\underline{u}$ is not trivial for $\lambda$ sufficiently large. To this end, let $t_{0}>1$ be a fixed real and $\Omega_{0} \subset \Omega$ be a nonempty open subset. Therefore, we infer that there is an element $\underline{v} \in C_{0}^{\infty}(\Omega) \subset E$ so that $\underline{v}=t_{0}$, for all $x \in \overline{\Omega_{0}}$ and $\underline{v} \in\left[0, t_{0}\right]$ for every $x \in \Omega \backslash \Omega_{0}$. We have the following

$$
\begin{aligned}
J_{\lambda, \mu}(\underline{v}) & =\Lambda(\underline{v})-\lambda \int_{\Omega} \frac{V(x)}{m(x)}|\underline{v}|^{m(x)} d x+\mu \int_{\Omega} G(x, \underline{v}) d x \\
& \leq L_{0}+k(\mu)-\frac{\lambda}{m^{+}} \int_{\Omega} V(x)|\underline{v}|^{m(x)} d x \\
& \leq L(\mu)-\frac{\lambda}{m^{+}} \int_{\Omega_{0}} V(x)\left|t_{0}\right|^{m(x)} d x \\
& \leq L(\mu)-\frac{\lambda}{m^{+}} C^{m^{-}}|V(x)|_{r(x)} t_{0}^{m^{-}} d x
\end{aligned}
$$

where $L(\mu)$ is a positive constant. Thus, there exist $\lambda^{* *}$, such that $J_{\lambda, \mu}(\underline{v})<0$ for any $\lambda \in\left[\lambda^{* *}, \infty\right)$. This, together with the fact that $\underline{u} \in E$ is global minimizer of $J_{\lambda, \mu}$, gives us $J_{\lambda, \mu}(\underline{u})<0$ for any $\lambda \in\left[\lambda^{* *}, \infty\right)$. In other words, $\underline{u}$ is a nontrivial weak solution of our problem for $\lambda$ sufficiently large, and this completes the proof of Theorem 3.9. 


\section{References}

[1] D.R. Adams and L.I. Hedberg, Function Spaces and Potential Theory, Grundlehren der Mathematischen Wissenschaften (Fundamental Principles of Mathematical Sciences), Springer-Verlag, Berlin, vol. 314, (1996).

[2] R. Adams, Sobolev Spaces, Academic Press, New York, (1975).

[3] O. Allegue and M. Bezzarga, Three solutions for a class of quasilinear elliptic systems in Orlicz-Sobolev spaces, Complex Variables and Elliptic Equations, 58(9), 1-13, (2012).

[4] M. Avci and K. Suslu, On a robin problem in Orlicz-Sobolev spaces, TWMS J. App. Eng. Math. (2017).

[5] J. Chabrowski and Y. Fu, Existence of solutions for $p(x)$-Laplacian problems on a bounded domain, J. Math. Anal. Appl. 306, 604-618, (2005).

[6] Y. Chen, S. Levine, and M. Rao, Variable exponent, linear growth functionals in image processing, SIAM J. Appl. Math. 66, 1383-1406, (2006).

[7] Ph. Clément, B. de Pagter, G. Sweers and F. de Thélin, Existence of solutions to a semilinear elliptic system through Orlicz-Sobolev spaces, Mediterr. J. Math. 241-267, (2004).

[8] Ph. Clément, M. García-Huidobro, R. Manásevich and K. Schmitt, Mountain pass type solutions for quasilinear elliptic equations, Calc. Var. Partial Differential Equations 11, 33-62, (2000).

[9] X. L. Fan, Q. Zhang and D. Zhao, Eigenvalues for $p(x)$-Laplacian Dirichlet problem, Nonlinear Anal. 52, 1843-1852, (2003).

[10] Fang, F. and Tan, Z., Existence and Multiplicity of Solutions for a Class of Quasilinear Elliptic Equations: An OrliczSobolev Setting, J. Math. Anal. Appl. 389, 420-428, (2012).

[11] X. Fan, J. Shen, and D. Zhao, Sobolev embedding theorems for spaces $W^{k, p(x)}(\Omega)$, J. Math. Anal. Appl. 262, 749-760, (2001).

[12] X. L. Fan and D. Zhao, On the spaces $L^{p(x)}(\Omega)$ and $W^{m, p(x)}(\Omega)$, J. Math. Anal. Appl. 263, 424-446, (2001).

[13] M. Garciá-Huidobro, V.K. Le, R. Manásevich and K. Schmitt, On principal eigenvalues for quasilinear elliptic differential operators: An Orlicz-Sobolev space setting, Nonlinear Differential Equations Appl. (NoDEA) 6, 207-225, (1999).

[14] J. P. Gossez, Nonlinear elliptic boundary value problems for equations with rapidly (or slowly) increasing coefficients, Trans. Amer. Math. Soc. 190, 163-205, (1974).

[15] M. Hsini, N. Irzi and KH. kefi, Nonhomogeneous $p(x)$-Laplacian Steklov problem with weights, Complex Variables and Elliptic Equations, 1-16, DOI: 10.1080/17476933.2019.1597070, (2019).

[16] S. Heidarkhani, G. Caristi and M. Ferrara, Perturbed Kirchhof-type Neumann problems in Orlicz-Sobolev spaces, Comput. Math. Appl. 71, 2008-2019, (2016).

[17] KH. kefi, $p(x)$-laplacian with indefinite weight, proceedings of the american mathematical society, Vol. 139, 12, 4351-4360, (2011).

[18] M. Mihăilescu and V. Rădulescu, Neumann problems associated to nonhomogeneous differential operators in Orlicz-Sobolev spaces, Ann. Inst. Fourier, 58, 2087-2111, (2008).

[19] M. Miháilescu and D. Repovś, Multiple solutions for a nonlinear and non-homogeneous problem in Orlicz-Sobolev spaces, Appl. Math. Comput. 217, 6624-6632, (2011).

[20] M. Miháilescu and V. Rádulescu, Eigenvalue problems associated to nonhomogeneous differential operators in Orlicz-Sobolev spaces, Anal. Appl. 6 (1), 1-16, (2008).

[21] I. L. Stăncut and I. D. Stircu, Eigenvalue problems for anisotropic equations involving a potential on Orlicz-Sobolev type spaces, Opuscula Math. 36, no. 1, 81-101, (2016). 
[22] M. Struwe, Variational Methods, Applications to Nonlinear Partial Differential Equations and Hamiltonian Systems, Springer, Heidelberg, (1996).

[23] V.D. Rădulescu, Nonlinear elliptic equations with variable exponent: old and new, Nonlinear Anal. 121, 336-369, (2015).

[24] V.D. Rădulescu and D.D. Repovš, Partial Differential Equations with Variable Exponents: Variational Methods and Qualitative Analysis, Monographs and Research Notes in Mathematics, Taylor \& Francis, Chapman and Hall/CRC, (2015).

[25] M.M. Rao and Z.D. Ren, Theory of Orlicz Spaces, Marcel Dekker, Inc., New York, (1991).

[26] M. Ruzicka, Electrorheological Fluids: Modeling and Mathematical Theory, Springer, Berlin, (2000).

[27] P. Rabinowitz, Minimax Methods in Critical Point Theory with Applications to Differential Equations, Expository Lectures from the CBMS Regional Conference held at the University of Miami, American Mathematical Society, Providence, RI, (1984). 